\title{
Vesicular protein transport
}

\section{P Schu}

Correction to: The Pharmacogenomics Journal (2001) 1: 262-271

The Pharmacogenomics Journal (2002) 2, 69. DOI: 10.1038/sj/tpj/6500097

Due to a typesetting error, part of the text on page 263 of this article was unreadable. The missing text is reproduced below.

There are two coat protein complexes, COP-I and COP-II, which mediate transport between the endoplasmic reticulum and the Golgi as well as within the Golgi. ${ }^{1}$ However there are 10 coat protein complexes and proteins known, which are involved in protein sorting between the trans-Golgi network and the plasma membrane. The family of heterotetrameric adaptor protein complexes mediating vescular protein transport in post-Golgi compartments and the ER/Golgi COP complexes are phylogenetically related. ${ }^{2}$ They are all ubiquitously expressed in mammals and many of these also exist as tissuespecific isoforms encoded by different genes or generated by alternative splicing. Formation of transport vesicles and protein sorting by coat proteins is compartment specific. The AP-2 complex for example mediates vesicle formation and protein sorting only at the plasma membrane.

Accessory proteins regulating vesicle budding and formation, vesicle transport and vesicle fusion with the proper target membrane bind to specialized domains of the coat proteins. They also interact with each other generating a complex regulatory network we are just beginning to understand. Lipid metabolism is required for proper protein sorting and phosphatidylinositol phosphorylation and dephosphorylation is a key regulatory element. Coat proteins and accessory proteins specifically bind to these phosphoinositides. Finally there is a family of proteins ensuring fusion of the vesicle with the proper target membrane. Some of these are membrane proteins and are part of the protein coat, whereas others cycle between the membrane and the cytoplasm regulated by protein phosphorylation and GTPase cycles. 